\title{
Free gracilis transfer for elbow flexion for pan brachial plexus injury
}

\author{
Mathangasinghe $\mathrm{Y}^{1}$, Rajapakse $\mathrm{Y} \mathrm{N}^{1}$, Rajapakse G K ${ }^{2}$, Beneragama T $\mathrm{S}^{3}$ \\ ${ }^{1}$ Department of Anatomy, Faculty of Medicine, University of Colombo, Sri Lanka \\ ${ }^{2}$ Department of Plastic and Reconstructive Surgery, Military Hospital, Sri Lanka \\ ${ }^{3}$ National Hospital of Sri Lanka
}

Keywords: Gracilis muscle; free tissue flaps; brachial plexus

\section{Introduction}

Global [Pan] Brachial Plexus Injuries [BPI] cause severe compromise of the upper limb functions, hence a significant disability [1]. Reinstating elbow flexion may contribute much towards restoring the function of the upper limb making it a helping hand [2]. Here, we present a case of restoration of elbow flexion in a patient with a global BPI using free functioning gracilis transfer. To our knowledge, this is the first report of a successful free gracilis transfer performed in Sri Lanka for a pan brachial plexus injury.

\section{Case presentation}

A 23-year-old male presented with a left-sided global BPI [C5 to T1] following a motorcycle accident. On clinical examination power of his shoulder, elbow and wrist movements were Medical Research Council [MRC] grading M0. Nerve conduction test confirmed severe global BPI [root evulsion type] involving both preganglionic and postganglionic segments. Three years after the initial injury he underwent nerve banking with the phrenic nerve as the donor motor nerve. A $10 \mathrm{~cm}$ sural nerve graft was used coaptating with the phrenic nerve to reach the mid-arm at the same time. One year after the nerve banking procedure, he underwent neuroma exploration [Supplementary Figure 1] and free functional gracilis transfer for elbow flexion. During this procedure, free functioning gracilis myocutaneous flap was harvested with its nerve supply [Supplementary Figure 2]. Branches of the brachial artery and brachial vein were anastomosed end-to-side with the recipient's vessels. Nerve end neuroma was taken for biopsy to confirm active axons. Neuroma was excised and was coaptated with the motor nerve to the gracilis. The proximal end of the gracilis was anchored to the coracoid process [Figure 1] and its distal end was attached to the biceps tendon [Figure 2]. Skin defect was closed incorporating the skin island of the gracilis which was used for monitoring of the flap during the postop time. A back slab was applied with elbow flexion of 90 degrees. Gentle

Correspondence: Yasith Mathangasinghe

E-mail: yasith@anat.cmb.ac.lk

(iD) https://orcid.org/0000-0003-4641-5642

Received: 24-05-2021 Accepted: 02-07-2021

DOI: http://doi.org/10.4038/sljs.v39i3.8706 active mobilization was started six weeks after the surgery, whereas weight-bearing exercises to strengthen the motor power of the biceps were started in 8 months. Muscle power of M3 was achieved after an 8-months follow-up, and the pain measured on a visual analogue scale was zero.

\section{Discussion}

The surgical management of brachial plexus injuries is challenging in developing countries due to the sparsity of resources and expertise. Surgical interventions for BPIs are indicated if $[\mathrm{A}]$ there is no spontaneous recovery following the first few weeks of trauma, [B] when discrete nerve injuries are expected/confirmed [eg. penetrating trauma] or [C] associated vascular injury. When feasible, early/immediate surgical repair is recommended over delayed repair since the former allows end-to-end suture or short grafting, which is often difficult when the nerve retracts with time. Nerve transfer procedures with contralateral $\mathrm{C} 7$, spinal accessory nerve, phrenic nerve, motor nerves of the cervical plexus and intercostal nerves have been successfully carried out within the first year of the injury [3]. However, the majority of the late presenters of total BPI benefits more by free functioning muscle transfer procedures compared to nerve repair for restoring elbow flexion [3], particularly when the denervation time is more than one year [2]. The phrenic nerve has a very high density of motor nerve fibres per unit area of crosssection, making it a good choice as a donor nerve [4]. Compromising of cardiopulmonary functions can occur as a result of the utilization of the phrenic nerve as a motor neurotizer, however, long-term follow-up data are sparse in the literature. Sacrificing the phrenic nerve is known to reduce vital capacity by approximately $10 \%$, however, this may not lead to clinically significant compromising of the respiratory functions [5]. In our case, we did not observe clinically detectable pulmonary or cardiac complications during one year of follow-up. Nevertheless, the phrenic nerve should be not considered as a donor nerve in infants and children because of the high respiratory demand [3]. Furthermore, obtaining the full length of the phrenic nerve requires thoracoscopic procedures, which may increase the morbidity of patients. Hence only a part of the nerve in the vicinity of the scalenus anterior is mobilized during this procedure. If the donor's nerve fails to innervate the recipient's muscle, it 


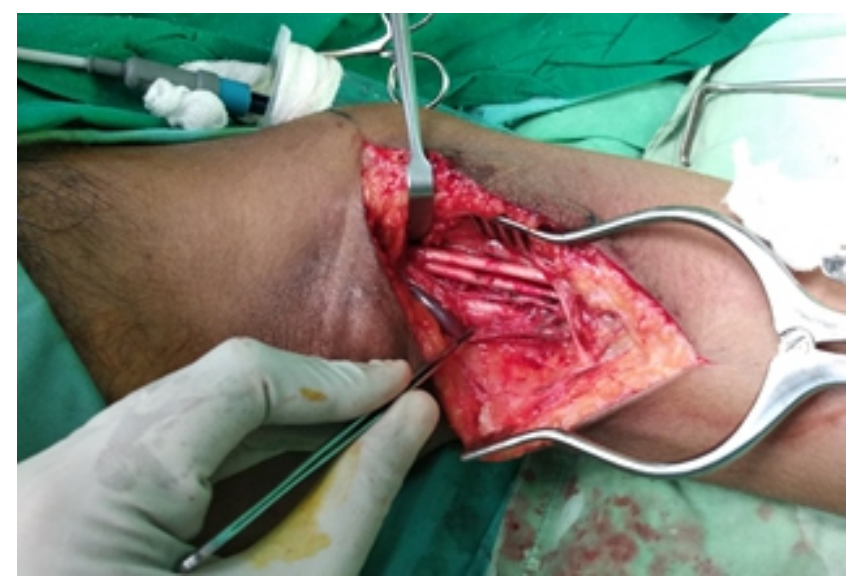

Figure 1. Proximal end of the gracilis myocutaneous flap anchored to the coracoid process

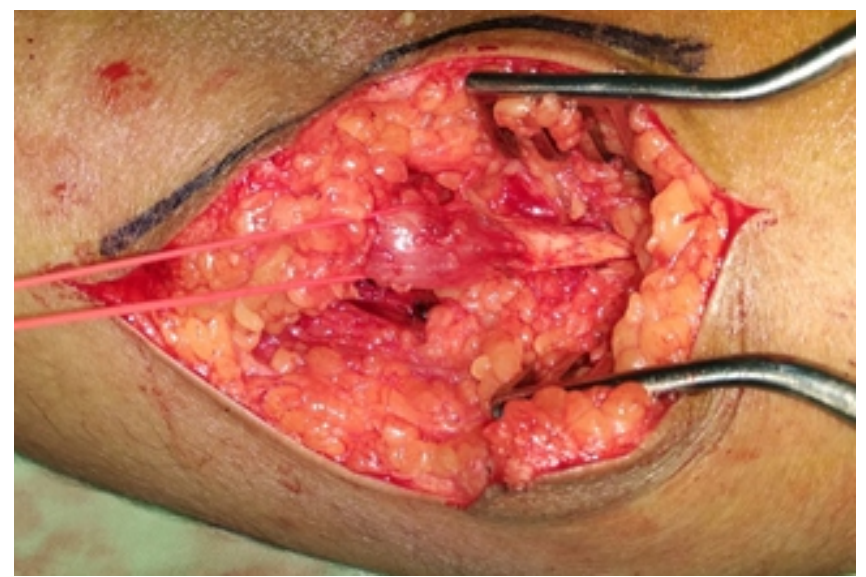

Figure 2. Distal end of the gracilis myocutaneous flap attached to the tendon of the biceps

subsequently undergoes atrophy. The rationale of nerve banking is to ensure the viability of the donor's nerve before initiating muscle transfer procedure and to achieve to appropriate length without tension. As in our case, axonal sprouting of the donor's nerve can be identified preoperatively by the presence of a neuroma which can be palpated subcutaneously with a positive Tinel sign and can be confirmed subsequently by histological examination. Yang et al, analysed 47 cases of functioning gracilis free transfer for global BPI using spinal accessory $[n=45]$ and phrenic $[n=2]$ nerve grafts from 1999 to 2016 [2]. Good elbow flexion, functional recovery and quality of life were observed in most of the participants of this study. In a Chinese clinical trial, less power of elbow flexion was achieved when the phrenic nerve was grafted to the musculocutaneous nerve directly or through a sural nerve graft in comparison to the partial transfer of median or ulnar nerves to the musculocutaneous nerve [6]. In our case, we were able to achieve an MRC grade 3 muscle power within 8 months postoperatively, which is considered as a clinically useful strength of elbow flexion [3].

All authors disclose no conflict of interest. The study was conducted in accordance with the ethical standards of the relevant institutional or national ethics committee and the Helsinki Declaration of 1975, as revised in 2000 .

\section{References}

1. Kaiser R, Waldauf P, Ullas G, Krajcová A. Epidemiology, etiology, and types of severe adult brachial plexus injuries requiring surgical repair: systematic review and meta-analysis. Neurosurgical review. 2018:1-10. Doi: 10.1007/s10143-0181009-2

2. Yang Y, Yang J-T, Fu G, et al. Functioning free gracilis transfer to reconstruct elbow flexion and quality of life in global brachial plexus injured patients. Scientific reports. 2016;6:22479. Doi: 10.1038/srep22479 [2016].

3. Hoang D, Chen VW, Seruya M. Recovery of elbow flexion after nerve reconstruction versus free functional muscle transfer for late, traumatic brachial plexus palsy: a systematic review. Plastic and reconstructive surgery. 2018;141[4]:949-959. Doi: 10.1097/PRS.0000000000004229

4. Viterbo F, Franciosi LF, Palhares A. Nerve graftings and end-toside neurorrhaphies connecting the phrenic nerve to the brachial plexus. Plastic and reconstructive surgery. 1995;96[2]:494. Doi: 10.1097/00006534-199508000-00054

5. Luedemann W, Hamm M, Blömer U, Samii M, Tatagiba M. Brachial plexus neurotization with donor phrenic nerves and its effect on pulmonary function. Journal of neurosurgery. 2002;96[3]:523-526. Doi: 10.3171/jns.2002.96.3.0523

6. Hou Z, Xu Z. Nerve transfer for treatment of brachial plexus injury: comparison study between the transfer of partial median and ulnar nerves and that of phrenic and spinal accessary nerves. Chinese journal of traumatology.

\section{Learning Points:}

- The late presenters of total BPI benefit more by free functioning muscle transfer procedures compared to nerve repair for restoring elbow flexion

- Phrenic nerve has a very high density of motor nerve fibres per unit area of cross-section, making it a good choice as a donor's nerve

- The rationale of nerve banking is to ensure the viability of the donor's nerve before initiating muscle transfer procedure and to achieve to appropriate length without tension 